\title{
Review of the Clinical Decision-Making Skills in Undergraduate Nursing Students
}

\section{Jefferson Garcia Guerrero*}

Fakeeh College for Medical Sciences, Saudi Arabia

*Corresponding author: Jefferson Garcia Guerrero, Fakeeh College for Medical Sciences, Saudi Arabia, Tel: +966570302970; E-mail: jgguerrero@fcms.edu.sa Received date: Feb 12, 2019; Accepted date: Mar 07, 2019; Published date: Mar 14, 2019

Copyright: @ 2019 Guerrero JG. This is an open-access article distributed under the terms of the Creative Commons Attribution License, which permits unrestricted use, distribution, and reproduction in any medium, provided the original author and source are credited.

\author{
Abstract \\ Objective: The intention of this systematic literature review is to describe and explore the existing eminence of \\ research evidence about clinical decision-making skills in undergraduate nursing students.
}

Methods: A review was conducted to determine the current knowledge approximately the chosen topic. PubMed and Science Direct online databases or systematic engine search were utilized to obtain the research articles relevant to this systematic review. The data consisted of 46 research articles about clinical decision-making skills using inclusion and exclusion criteria. The researcher used content and thematic analysis as a qualitative approach in reviewing the articles. Data analysis was implemented from November 10, 2018 to January 21, 2019.

Results: This systematic literature review revealed three major themes emerged in this study. Such major theme includes pre-requisite skills, clinical decision making skills and clinical judgment. The first theme emerged is the prerequisite skills. Sixteen studies have cited pre-requisite skills in clinical decision-making. Under pre-requisite skills, the following sub-themes emerged which includes knowledge, skills, self-confidence, and self-efficacy. The second theme emerged is the clinical decision-making skills. In here, twenty-four studies have supported that decisionmaking is an important clinical skill for undergraduate nursing students. Last major theme emerged is the nursing clinical judgment. Under this theme, eleven studies have supported nursing clinical judgment. Three sub-themes were identified that influence nursing students' clinical judgment. These include reflection, the learning environment and clinical teachers.

Conclusion: In general, the need to assist undergraduate nursing students in anticipating and exercising prerequisite skills, clinical decision making and professional nursing judgment is needed in their clinical placement setting.

Keywords: Nursing students; Pre-requisite skills; Clinical decisionmaking skills; Nursing clinical judgment; Clinical placement

\section{Introduction}

Healthcare field nowadays, the nurse is confronted with gradually multifarious disputes and circumstances. Currently, the decisionmaking fragment of problem-solving has developed progressively complicated and demands critical thinking [1]. Over the last 15 years, the study showed that decision-making skills are recurrently and significantly deteriorating among nurses and nursing students [2]. Studies even cited that graduates are incapable to display seemly clinical decision-making skills in clinical areas [3]. Numerous nursing students reported a deficiency of vigilance and competency with respect to their capacity to achieve nursing responsibilities [4]. In this connection, nurse educators have recognized that several nursing students have inconvenience in performing decision-making in clinical practice [5]. Thus, caring for patients requires prompt and effective decision-making abilities [4]

Critical thinking is a multidimensional skill, a cognitive or mental process or set of procedures. It entangles intellectual and persistent, logical, philosophical, sensible, an outcome-directed view grounded on a body of knowledge, as well as consideration of all obtainable information and mindsets [6]. Thus, student nurses need good clinical reason skills because deprived clinical reasoning skills can prime to failure-to competently care worsening patients [7].

On the other hand, clinical decision-making is a primary part of nursing practice [8]. Decision-making is an ultimate skill necessitated and recognized by the Nursing Council and should be succeeded in pre-registration nursing programs. Frequently, students do not acknowledge that voluminous of the undertakings they accomplish on clinical placement engage decision-making skills. Thus, nursing students must demonstrate their decision-making skills in clinical placement [9].

There had been limited studies about the capability to utilize information for clinical nursing decision-making [2]. Also, little research has been conducted that examines ways to recuperate the clinical decision-making capability of nursing students [10]. This prompted the researcher to develop a research paper entitled "A systematic review of clinical decision making and critical thinking skills among undergraduate nursing students" that will examine and explore the current status of research evidence about the use of decision making and critical thinking among nursing students in the clinical area. 
Page 2 of 5

\section{Search Methods}

A review of the literature was undertaken using a systematic approach. A review was conducted to determine the current knowledge about the clinical decision-making skills among nursing students. This systematic review was conceded subsequently by a wideranging literature exploration exhausting electronic databases such as PubMed, CINAHL, and Science Direct. Online databases or engine search were utilized to obtain the research articles relevant to this systematic review and also to enable high search sensitivity. Search terms and subject headings were identified and searched. The selected articles were authored by researchers from England, Turkey, Brazil, Australia, Greece, USA, Canada, Korea, UK, Sweden, Spain and Iran.

\section{Search outcomes}

Eighty-two relevant research articles were retrieved that were published from the years 2009 to 2019. All obtained research articles are about clinical decision-making skills among nursing students. Thus, 31 eligible studies were identified. Inclusion criteria of the study include the following: a) All selected articles must be published in English language; b) All articles must be published in peer-reviewed journals; c) All articles must be published from at least 10 years from now (2009-2019); The researcher decided to include a ten-year period of journal publication to be sufficient enough to make a comprehensive literature search. d) All articles must include undergraduate nursing students as the study population; e) All articles must contain at least one of the following keywords clinical decision-making skills, nursing students, clinical placement; and lastly f) all articles must either be descriptive design, correlational study, observational study, exploratory study, phenomenological qualitative study or mixed method. Thus, all studies were incorporated in this review if they convened the inclusion criteria.

On the other hand, Exclusion criteria are criteria which may not allow any articles to be included in the study. The exclusion criteria include the following: a) All case studies and case reports were omitted due to excessive conceivable biases; b) All experimental designs (RCT, quasi studies) were also excluded; and c) All articles with secondary data and not report primary data like meta-analysis, meta-synthesis, and integrative literature review.

\section{Data extraction}

Key information, title review, research design, sample participants, research focus, and study outcomes were extricated from the preferred articles. Data were extracted by two reviewers to perform a quality assessment and to evaluate the quality of studies. The primary researcher self-sufficiently fulfilled abstraction form while the second researcher double-checked the first reviewer's entry for verification, accuracy, clarity and completion purposes. Data analyses were performed from November 10, 2018 to January 21, 2019.

\section{Data synthesis}

The researcher used content analysis and thematic analysis as qualitative approaches in reviewing the articles. Content analysis is a research method for studying social phenomena using the available documents, artifact, and literature to examine pattern in a systematic manner/way. On the other hand, thematic analysis was performed to integrate the information presented in this review.

\section{Flowchart of Literature Search (PRISMA)}

The researcher has utilized PRISMA as an evidence-based minimum set of items for reporting in systematic reviews. PRISMA is used as a basis for reporting and critical appraisal of published systematic reviews of other types of research. It aims to help authors improve the reporting of systematic reviews.

- 1,332 research articles were identified through electronic database search (593 articles were from PubMed, and 739 were from ScienceDirect).

- Afterward, 158 duplicate studies were excluded.

- Next, 1174 research articles were identified for title review (541 articles removed).

- On the other hand, 633 research articles were identified for abstract review (547 research articles were excluded).

- 86 articles were included in the in-depth review using full manuscript assessment (research design, sample participants, research focus, and study outcomes) using inclusion and exclusion criteria (40 articles remained excepted) (Figure 1).

- Lastly, a total of 46 research articles were included for systematic review.

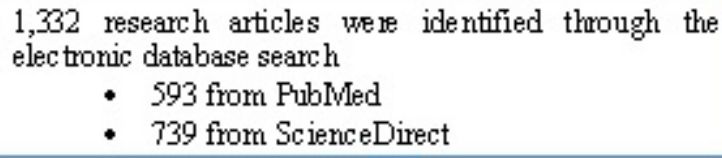

158 duplicate research articles we re excluded.

1174 research articles were identified for title review (541 articles excluded).

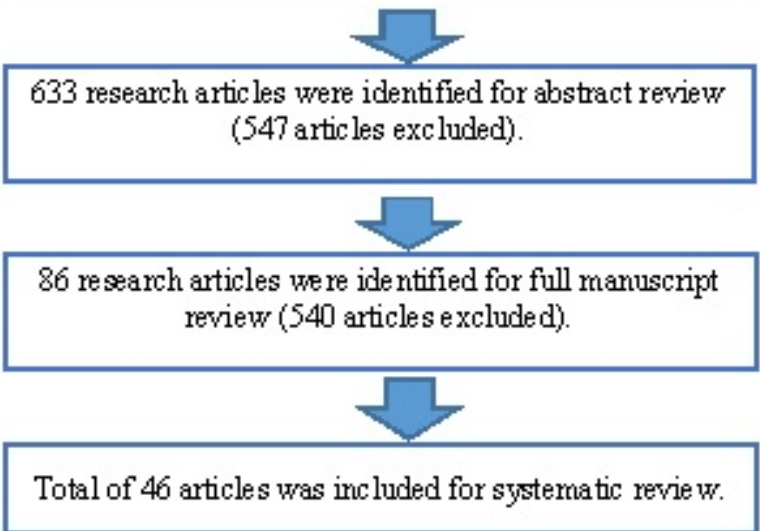

Figure 1: Flowchart of literature search (PRISMA).

\section{Results and Discussion}

This systematic review revealed at least three general themes emerged in this study. Such major themes include pre-requisite skills, 
clinical decision-making skill, and clinical judgment. The first theme talks about the pre-requisite skills that are indispensable to sustain clinical decision-making. The second theme is the main highlight which discusses the importance and relevance of clinical decisionmaking skill in undergraduate nursing students. The last theme is clinical judgment the outcome as a result of the use of decision-making in the clinical practice settings.

\section{Pre-requisite skills for clinical decision-making}

Based on the result of this systematic review, sixteen studies have cited pre-requisite skills in clinical decision-making. Accordingly, prerequisite skills are fundamental skills that are necessary for a nursing student to perform clinical decision-making abilities. The following sub-themes under pre-requisite skill emerged, namely: theoretical knowledge, clinical technical skills, self-confidence, and self-efficacy.

First basic skill is theoretical knowledge. Seven studies have shown that nursing students' prior knowledge influences and is necessary for decision-making in the clinical setting [11-17]. Knowledge as an imperative and dominant feature of student nurses is important in the practice settings [12]. Thus, it can be stated that the professional and theoretical knowledge is a non-technical skill which is essential in critical thinking, clinical reasoning, and decision-making process for undergraduate nursing students [13,14]. Moreover, nursing schools must assist students in the integration and application of their theoretical knowledge [15]. Because uncertainty, lack of knowledge, inadequate information, untimely diagnosis and disappointment to deliberate selections when concerning for patients is possible to affect and prime to low quality decisions [16].

Second pre-requisite skill is the clinical technical skill of the student nurse. Three studies have demonstrated that nursing students' clinical skill is necessary for decision-making and clinical reasoning in the clinical setting [17-20]. Nursing students must develop his nursing technical skills competency to be best used in the clinical practice setting. Moreover, nursing schools must assist students in the integration and application of their clinical skills in the clinical areas [15].

Last pre-requisite skill is self-efficacy. Self-efficacy is defined as one's ability to succeed in specific situation necessary to accomplish a task. Students must develop self-efficacy in their own clinical abilities [21]. One study has cited that graduate student nurses are incapable to exhibit appropriate clinical decision-making skills because of truncated self-efficacy [3]. Thus, undergraduate nursing students must practice self-efficacy to be effective, efficient and productive in eliciting clinical reasoning in stressful clinical situations [19].

In general, knowledge, skills, self-confidence, and self-efficacy are pre-requisite skills needed to perform clinical decision making among undergraduate nursing students decision-making abilities. The following sub-themes under pre-requisite skill emerged, namely: theoretical knowledge, clinical technical skills, self-confidence, and self-efficacy.

First basic skill is theoretical knowledge. Seven studies have shown that nursing students' prior knowledge influences and is necessary for decision-making in the clinical setting [14-17]. Knowledge as an imperative and dominant feature of student nurses is important in the practice settings [12]. Thus, it can be stated that the professional and theoretical knowledge is a non-technical skill which is essential in critical thinking, clinical reasoning, and decision-making process for undergraduate nursing students [13,14]. Moreover, nursing schools must assist students in the integration and application of their theoretical knowledge [15]. Because uncertainty, lack of knowledge, inadequate information, untimely diagnosis and disappointment to deliberate selections when concerning for patients is possible to affect and prime to low quality decisions [16].

Second pre-requisite skill is the clinical technical skill of the student nurse. Three studies have demonstrated that nursing students' clinical skill is necessary for decision-making and clinical reasoning in the clinical setting $[14,15,17]$. Nursing students must develop his nursing technical skills competency to be best used in the clinical practice setting. Moreover, nursing schools must assist students in the integration and application of their clinical skills in the clinical areas [15].

Last pre-requisite skill is self-efficacy. Self-efficacy is defined as one's ability to succeed in specific situation necessary to accomplish a task. Students must develop self-efficacy in their own clinical abilities [21]. One study has cited that graduate student nurses are incapable to exhibit appropriate clinical decision-making skills because of truncated self-efficacy [3]. Thus, undergraduate nursing students must practice self-efficacy to be effective, efficient and productive in eliciting clinical reasoning in stressful clinical situations [19].

In general, knowledge, skills, self-confidence, and self-efficacy are pre-requisite skills needed to perform clinical decision making among undergraduate nursing students.

\section{Decision-making as an important clinical skill for undergraduate nursing students}

Based on the result of the systematic review, twenty-four studies have supported that decision-making is a significant clinical skill for undergraduate nursing students. Accordingly, decision making skill is a high-level skill that is difficult to assess. Patient care compels timely and successful decision-making capabilities [4]. Such skill is essential to recognize and intervene the patient whose state is declining [22-27]. One study cited that new graduate nurses are incompetent to establish suitable clinical decision-making skills [3]. The need to exhibit clinical decision-making skill is expected to entry-level graduate nurse [14]. Habitually, students do not acknowledge that countless of the responsibilities they play on clinical placement encompass decisionmaking skills [9].

Students must be confident in the utilization of clinical decisionmaking [27]. This can be achieved by attaining basic competence in healthcare decision-making of the students during their clinical practicum $[28,29]$. Furthermore, clinical decision making is a keystone skill and essential for undergraduate student nurses because it Clinical decision-making skill enables the nursing student to complete nursing interventions and provide nursing care aligned with course learning objectives [13,18,23,25,30,31].

In line with this, decision making is a central skill necessitated and identified by the Nursing Council and must be achieved in preregistration nursing programs [9]. Nursing schools and nurse educators must help nursing students enhance learning clinical decision-making [3]. This can be achieved by developing cognitive strategies which can help reduce errors in clinical decision-making [14]. Also, student nurses must be provided with a multitude of case situations where clinical decisions are built those impact patient outcomes [32]. Therefore, the need to assist students in anticipating and exercising decision making is needed [14]. Also, nursing students 
Page 4 of 5

must demonstrate their decision-making skills in clinical placement [9]. Students' clinical decision-making is a continuous development during the whole clinical task [33].

In addition, decision-making is important in the management of rare complex crisis situations. The need to foster student ability to use clinical decision-making to complex, multi-contextual challenging, and ambiguous clinical scenarios [34]. Likewise, the capacity to judiciously appraise patient care circumstances is necessary for decision-making skills [35].

Decision making skill is necessary to provide holistic care to their patients [36]. Decision-making is a non-technical skill which is a fundamental requirement for health professional graduates to ensure secure and proficient practice in the clinical setting [37]. Thus, clinical decision-making is an intricate endeavor that is serious to patient safety [38]. Furthermore, it is important for nursing students because it affects patient outcomes and can influence health outcomes $[18,35,39]$. Thus, undergraduate nursing students must gain sound clinical decisions because decision-making is an essential part of the nursing practice $[8,40]$.

\section{Nursing clinical judgment}

Based on the result of the systematic review, eleven studies have similar studies about nursing clinical judgment. Accordingly, clinical judgment is the outcome or result of the use of decision-making in the clinical practice settings. Clinical judgment is indispensable for clinical decision making [41].

Nursing clinical judgment is a core competency that must be developed in nursing education [42]. Pre-licensure nursing students must develop their capacity to perform clinical judgment [25]. Judgments in decision-making are basic student nurse competencies used during clinical practice. The need for a shared understanding of judgment criteria is also important [29]. Clinical judgment embodies how student nurses utilize their comprehension of the patient to produce, measure, and arrange patient care discretions [42].

Three sub-themes were identified that influence nursing students' clinical judgment. These include reflection, the learning environment and clinical teachers [41]. First sub-theme is a reflection. It is the ability to reflect on critical incidents or practice events [43]. Learning reflection is important in promoting clinical decision-making because it has a role in broadening students' knowledge and understand their beliefs and experiences [44]. Second is the learning environment. This refers to the suitable informative circumstance and a harmless psychological environment [41]. Curricular changes on nursing students' clinical learning outcomes resulted in improved clinical judgment [24]. Third sub-theme is a clinical teacher. The clinical teacher is the individual or professional who uses instructional strategies to establish clinical judgment in nursing students [41]. The need to promote student skill to employ clinical judgment in a complex, multi-contextual challenging, and ambiguous clinical scenarios is imperative [45].

Thus, enhancing clinical judgment abilities of nursing students can help improves health care quality, and can influence health outcomes $[11,39,41]$. Also, the need to develop clinical judgment in Bachelor of Science nursing students aims to deliver high-quality, competent, and considerate nursing care to patients and their families [46].

\section{Conclusion and Recommendation}

In general, the need to assist undergraduate nursing students in anticipating and exercising pre-requisite skills, clinical decisionmaking, and professional nursing judgment is needed in their clinical placement setting. Based on the result of the systematic review, one must elicit theoretical knowledge, technical skills, self-confidence, and self-efficacy as pre-requisite skills necessary to perform clinical decision making. Undergraduate nursing students must gain sound clinical decisions because clinical decision-making is an essential and imperative part of the nursing practice. Lastly, clinical judgment is the outcome or result of clinical decision-making skill. Under this theme, three sub-themes influence nursing students' clinical judgment. These include reflection, the learning environment and clinical teachers. Therefore, the need to assist students in anticipating and exercising decision making is needed. Also, future research is needed to gather information and research evidence approximately the importance of clinical decision-making skills to nursing students. Furthermore, the researcher advise future researcher to conduct similar study that will include other electronic database like ProQuest, EBSCO, and CINAHL to provide more detailed and comprehensive literature search.

\section{References}

1. Hinkle JL, Cheever KH (2018) Brunner and Suddarth's textbook of medical-surgical nursing. (14th edn), Lippincott Williams and Wilkins, Philadelphia.

2. Canova C, Brogiato G, Roveron G, Zanotti R (2016) Changes in decisionmaking among Italian nurses and nursing students over the last 15 years. J Clin Nurs 25: 811-818.

3. Jahanpour F, Sharif F, Salsali M, Kaveh MH, Williams LM (2010) Clinical decision-making in senior nursing students in Iran. Int J Nurs Pract 16: 595-602.

4. Lee JJ, Jeong HC, Kang KA, Kim YJ, Lee MN (2015) Development of a simulation scenario and evaluation checklist for patients with asthma in emergency care. Comput Inform Nurs 33: 546-554.

5. Sullivan AE (2012) Critical thinking in clinical nurse education: application of Paul's model of critical thinking. Nurse Educ Pract 12: 322-327.

6. Ignatavicius DD, Workman ML (2016) Medical-surgical nursing: Patientcentered collaborative care. (8th edn), St. Louis, Missouri: Elsevier.

7. Hoffman K, Dempsey J, Levett-Jones T, Noble D, Hickey N, et al. (2011) The design and implementation of an Interactive Computerised Decision Support Framework (ICDSF) as a strategy to improve nursing students' clinical reasoning skills. Nurse Educ Today 31: 587-594.

8. Wang Y, Chien WT, Twinn S (2012) An exploratory study on baccalaureate-prepared nurses' perceptions regarding clinical decisionmaking in mainland China. J Clin Nurs 21: 1706-1715.

9. Ness V, Duffy K, McCallum J, Price L (2010) Supporting and mentoring nursing students in practice. Nurs Stand 25: 41-46.

10. Laney SP, Keen C, Hall K (2012) The use of human patient simulators to enhance clinical decision-making of nursing students. Educ Health 25: 11-15.

11. Griffith L, Board M (2018) Influences on clinical decision-making during a community placement: reflections of a student nurse. Br J Community Nurs 23: 606-609.

12. Kaya H, Şenyuva E, Bodur G (2017) Developing critical thinking disposition and emotional intelligence of nursing students: a longitudinal research. Nurse Educ Today 48: 72-77.

13. Menezes SS, Corrêa CG, Silva Rde C, Cruz Dde A (2015) Clinical reasoning in undergraduate nursing education: a scoping review. Rev Esc Enferm USP 49: 1037-1044. 
14. Jeffrey K, Bourgeois S (2011) The effect of personal digital assistants in supporting the development of clinical reasoning in undergraduate nursing students: A systematic review. JBI Libr Syst Rev 9: 38-68.

15. Papathanasiou IV, Kleisiaris CF, Fradelos EC, Kakou K, Kourkouta L (2014) Critical thinking: the development of an essential skill for nursing students. Acta Inform Med 22: 283-286.

16. Bucknall TK, Forbes H, Phillips NM, Hewitt NA, Cooper S, et al. (2016) An analysis of nursing students' decision-making in teams during simulations of acute patient deterioration. J Adv Nurs 72: 2482-2494.

17. Ramos FR, Brehmer LC, Vargas MA, Trombetta AP, Silveira LR, et al. (2015) Ethical conflicts and the process of reflection in undergraduate nursing students in Brazil. Nurs Ethics 22: 428-439.

18. White KA, Fetter ME, Ruth-Sahd LA (2018) Extern programs promote confidence and reduce anxiety with clinical decision-making in nursing students. Nurse Educ.

19. Woda A, Hansen J, Paquette M, Topp R (2017) The impact of simulation sequencing on perceived clinical decision making. Nurse Educ Pract 26: 33-38.

20. Hart PL, Spiva L, Mareno N (2014) Psychometric properties of the clinical decision-making self-confidence scale. J Nurs Meas 22: 312-322.

21. Lewis R, Strachan A, Smith MM (2012) Is high fidelity simulation the most effective method for the development of non-technical skills in nursing? A review of the current evidence. Open Nurs J 6: 82-89.

22. White KA (2014) Development and validation of a tool to measure selfconfidence and anxiety in nursing students during clinical decision making. J Nurs Educ 53: 14-22.

23. Woda AA, Gruenke T, Alt-Gehrman P, Hansen J (2016) Nursing student perceptions regarding simulation experience sequencing. J Nurs Educ 55: 528-532.

24. Landeen J, Carr D, Culver K, Martin L, Matthew-Maich N, et al. (2016) The impact of curricular changes on BSCN students' clinical learning outcomes. Nurse Educ Pract 21: 51-58.

25. Bussard ME (2016) Self-reflection of video-recorded high-fidelity simulations and development of clinical judgment. J Nurs Educ 55 522-527.

26. Benham B, Hawley D (2015) The effectiveness of tools used to evaluate successful critical decision making skills for applicants to healthcare graduate educational programs: a systematic review. JBI Database System Rev Implement Rep 13: 231-275.

27. McCallum J, Duffy K, Hastie E, Ness V, Price L (2013) Developing nursing students' decision making skills: Are early warning scoring systems helpful? Nurs Educ Pract 13: 1-3.

28. Choi M, Lee H, Park JH (2018) Effects of using mobile device-based academic electronic medical records for clinical practicum by undergraduate nursing students: A quasi-experimental study. Nurs Educ Today 61: 112-119.

29. Burden S, Topping AE, O'Halloran C (2018) Mentor judgment and decision-making in the assessment of student nurse competence in practice: A mixed-methods study. J Adv Nurs 74: 1078-1089.
30. Andrew LA, Baxter PM (2019) Incorporating innovative simulation activities into campus lab to enhance skill competence and critical thinking of second-semester associate degree nursing students. Nurs Educ Perspect 40: 58-59.

31. Squirrell B, Hunt J (2018) A nursing student's reflective account of decision-making in a school nursing setting. Nurs Child Young People 30: 26-29.

32. Ellis $\mathrm{N}$ (2017) Decision making in practice: influences, management and reflection. Br J Nurs 26: 109-112.

33. Nilsson T, Lindstrom V (2016) Clinical decision-making described by Swedish prehospital emergency care nurse students: An exploratory study. Int Emerg Nurs 27: 46-50.

34. McMahon MA, Christopher KA (2011) Case study method and problembased learning: Utilizing the pedagogical model of progressive complexity in nursing education. Int J Nurs Educ Scholarsh 8: 22.

35. Murray K, McKenzie K, Kelleher M (2016) The evaluation of a framework for measuring the non-technical ward round skills of final year nursing students: An observational study. Nurs Educ Today 45: 87-90.

36. Khalili H (2015) Clinical simulation practise framework. Clin Teach 12: 32-36.

37. Peddle M, Mckenna L, Bearman M, Nestel D (2019) Development of non-technical skills through virtual patients for undergraduate nursing students: An exploratory study. Nurs Educ Today 73: 94-101.

38. Forbes H, Bucknall TK, Hutchinson AM (2016) Piloting the feasibility of head-mounted video technology to augment student feedback during simulated clinical decision-making: An observational design pilot study. Nurs Educ Today 39: 116-121.

39. Cereto M, Mayor S, Uttumchandani S, Gámez M, Campos A, et al. (2018) Cultural adaptation and validation of the Lasater Clinical Judgment Rubric in nursing students in Spain. Nurs Educ Today 64: 71-78.

40. Guhde J (2010) Using online exercises and patient simulation to improve students' clinical decision-making. Nurs Educ Perspect 31: 387-389.|

41. Pouralizadeh M, Khankeh H, Ebadi A, Dalvandi A (2017) Factors influencing nursing students' clinical judgment: A qualitative directed content analysis in an Iranian context. J Clin Diagn Res 11: JC01-JC04.

42. Hines CB, Wood FG (2016) Clinical judgment scripts as a strategy to foster clinical judgments. J Nurs Educ 55: 691-695.

43. Andersen E (2016) Enhancing the clinical reflective capacities of nursing students. Nurse Educ Pract 19: 31-35.

44. Edelen BG, Bell AA (2011) The role of analogy-guided learning experiences in enhancing students' clinical decision-making skills. J Nurs Educ 50: 453-460.

45. O'Rourke J, Zerwic J (2016) Measure of clinical decision-making abilities of nurse practitioner students. J Nurs Educ 55: 18-23.

46. Glynn DM (2012) Clinical judgment development using structured classroom reflective practice: a qualitative study. J Nurs Educ 51: 134-139. 